\title{
Combination therapy using three novel prolactin receptor antagonist-based fusion proteins effectively inhibits tumor recurrence and metastasis in HER2/neu transgenic mice
}

\author{
SETH TOMBLYN ${ }^{1,2}$, ALISON E.B. SPRINGS ${ }^{2}$, JOHN F. LANGENHEIM ${ }^{1,2}$ and WEN Y. CHEN ${ }^{1,2}$ \\ ${ }^{1}$ Department of Biological Sciences, Clemson University, Clemson, SC 29634; ${ }^{2}$ Oncology Research Institute, \\ Greenville Hospital System, Greenville, SC 29605, USA
}

Received November 6, 2008; Accepted December 30, 2008

DOI: 10.3892/ijo_00000242

\begin{abstract}
Previously, prolactin receptor antagonist (G129R)based fusion proteins were developed including G129R fusions with an angiogenesis inhibitor (endostatin), an immune system modulator (interleukin 2), and a modified truncated cytotoxin $\left(\mathrm{PE}_{38} \mathrm{KDEL}\right)$. Each fusion protein was designed to target the PRLR-positive cells via the G129R moiety and at the same time attack a hallmark common to cancer cells via the second moiety. In this study, we tested the efficacy of the three fusion proteins as a combination therapy in an aggressive but clinically relevant mouse tumor model. To test the feasibility and to optimize a treatment regimen, allografts of a mammary carcinoma cell line (McNeuA) derived from an MMTV-neu transgenic mouse were first used. Growth of the allografts was significantly retarded by regimens which combined all three fusion proteins. In addition, a significant increase in cytotoxic $\mathrm{CD}^{+} \mathrm{T}$ cells was observed within the tumors of the combination treated groups. After establishing the dosing regimen, two doses of cocktail treatment (low and high doses administered twice weekly) along with individual component controls were administered to female MMTV-neu transgenic mice after surgical removal of a naturally occurring tumor. The average tumor recurrence time was significantly delayed in both low and high combination treatment groups in comparison to the no treatment control group (34, 50 and 18 days, respectively).
\end{abstract}

Correspondence to: Dr Wen Y. Chen, Oncology Research Institute, Greenville Hospital System, 900 West Faris Road, Greenville, SC 29605, USA

E-mail: wchen@ghs.org

Abbreviations: EGF, epidermal growth factor; G129R, human PRLR antagonist; HER2, human epidermal growth factor receptor 2; PRL, prolactin; PRLR, PRL receptor

Key words: breast cancer, combination therapy, fusion protein, HER2, neu, prolactin receptor antagonist
The total number of lung metastases was also significantly decreased in both combination treatment groups. In conclusion, using G129R-based fusion proteins to target mammary carcinomas and to tackle multiple hallmarks of cancer at the same time was an effective strategy for treating HER2postive mammary cancer in this mouse tumor model.

\section{Introduction}

Hormones that are intimately involved in normal mammary gland development such as estrogens, epidermal growth factor (EGF) and prolactin (PRL) influence the initiation and/or progression of breast cancer. Hormonal imbalance is a major contributing factor for developing breast cancer. Studies have shown that PRL is expressed in the epithelium of human breast cancers and that PRL receptor (PRLR) levels are higher in a majority of benign and malignant mammary tumors than the surrounding normal tissue $(1,2)$. In addition, PRL and PRLR appear to have a role in both hormone responsive and hormone-independent breast cancers (3-5). For example, PRL has been shown to interact synergistically with estrogen and progesterone to promote cancerous growth; the receptors for these ligands are often co-expressed in primary breast cancers (3). Furthermore, PRL is able to transactivate HER2 through the activation of PRLR $(4,5)$. In accordance, anti-proliferative effects of tamoxifen (6) or trastuzumab (7) were enhanced when they were combined with a PRLR antagonist, G129R.

G129R was developed for anti-breast cancer by substituting Gly at position 129 of human (h) PRL with Arg as previously reported $(6,8)$. It has been demonstrated that G129R dosedependently inhibits human breast cancer cell proliferation (6); competitively blocks hPRL-induced Janus-activated kinase 2 (JAK2)/signal transducer and activator of transcription (STAT), Ras/Raf-1/MEK/mitogen-activated protein kinases (MAPKs), and phosphoinositide 3-kinase (PI3K)/3phosphoinositide-dependent protein kinase 1 (PDK1)/Akt signal transduction; and up-regulates TGF- $B$ and downregulates $b c l-2$ which in turn lead to the arrest of cell cycle progression and in some circumstances the induction of apoptosis (9-11). In an effort to generate mammary tissuetargeting therapeutics, dual-functional fusion proteins were generated which combined G129R with an angiogenesis 
inhibitor, G129R-Endostatin (12); an immune system modulator, G129R-Interleukin 2 (IL2) (13); or a modified truncated cytotoxin, G129R-PE ${ }_{40}$ KDEL (14). It was demonstrated that the G129R moieties of the fusion proteins retained the ability to bind to PRLR, thus directing the fusion proteins to PRLR rich mammary cells and competitively blocking hPRL-induced signal transduction (12-14). The second moiety of the fusion proteins have also been shown to retain proper function. G129R-Endostatin was as effective as endostatin alone at inhibiting tube formation and proliferation of HUVEC cells (12). G129R-IL2 was able to stimulate the proliferation of CTLL2 cells as effectively as IL2 (13). G129R-PE ${ }_{40}$ KDEL showed specificity towards breast cancer cells expressing the PRLR, inhibited protein synthesis, and induced cell death (14). Finally, both G129R-Endostatin and G129R-IL2 fusion proteins as a monotherapy were able to slow tumor growth in mice $(12,13)$.

In recent years, the focus on cancer therapy has shifted from traditional chemotherapy, which employs small molecules that target DNA synthesis and replication activities, to novel therapeutic agents, which target unique biomarkers of cancer cells. Therapeutics have been designed which exploit overexpressed receptors (such as HER2/neu), tumor oncoproteins (such as Bcr-Abl), or proteins associated with tumor angiogenesis (such as VEGF/VEGFR) (15-17). Another shift in the therapeutic paradigm has been the use of a combinational approach in the hope that each component in the combination therapy will target a different mechanism exploited by the cancer, thus maximizing the response. For example, the use of trastuzumab along with paclitaxel, cisplatin, or radiation has been shown to increase the survival rate by $\leq 50 \%$ (18). Imatinib mesylate, a small molecule Bcr-Abl inhibitor, sensitizes cancer cells to radiation (19). The use of a monoclonal antibody specific for the EGF receptor (EGFR), cetuximab, and a small molecular inhibitor of the EGFR, gefitinib, in combination show a shared complementary mode of action and a synergistic response in vivo $(20,21)$.

In the present study, a clinically relevant mouse tumor model was used to test the efficacy of a combination therapy composed of three fusion proteins, G129R-IL2, G129REndostatin and G129R-PE ${ }_{38}$ KDEL. A highly significant and dose-dependent delay in tumor recurrence and a reduction in lung metastases were observed in mice treated with the fusion protein combinations.

\section{Materials and methods}

Protein production. The $\mathrm{pET} 22 \mathrm{~b}(+)$ based plasmids containing cDNAs encoding G129R-PE ${ }_{38}$ KDEL, G129R-IL2 or G129REndostatin were transformed into Rossetta (DE3)pLysS competent $E$. coli cells (Novagen/EMD Biosciences, San Diego, CA). Protein expression, isolation of inclusion bodies, refolding, and purification protocols were reported previously $(6,12)$. The function of both moieties of each fusion protein was determined as described previously (12-14). To improve the yield of G129R-IL2, an unpaired Cys at position 125 of IL2 was substituted with an Ala. This substitution mutation does not affect the function of IL2 (22). To enhance the cytotoxicity of G129R-PE $\mathrm{F}_{40} \mathrm{KDEL}$, domain 1a of the toxin moiety was removed to create G129R-PE ${ }_{38}$ KDEL (23).
Transgenic mice. Breeding pairs of FVB/N-Tg(MMTVneu) 202Mul/J mice, homozygous for the unactivated form of rat erbB2/neu transgene under the control of the mouse mammary tumor promoter (MMTV-neu) (24) were obtained from Jackson Laboratory (Jackson Laboratory, Bar Harbor, Maine). The colony was expanded and housed in accordance with The Guide for the Care and Use of Laboratory Animals. To implant allografts or remove tumors, mice were anesthetized with $0.4 \mathrm{mg} / \mathrm{g}$ body weight of 2,2,2-tribromethanol (SigmaAldrich, St. Louis, MO) prepared in tert-amyl alcohol (Sigma-Aldrich) and saline.

$R T$-PCR. To confirm expression of mouse PRLR, mammary gland or tumor tissue of MMTV-neu transgenic mice were dissected and frozen until time of use. Approximately 50-100 mg of frozen tissue was homogenized in $1 \mathrm{ml}$ TRIzol (Invitrogen, Carlsbad, CA) on ice using a motorized homogenizer and processed according to the manufacturer's instructions to isolate RNA. RT-PCR was performed with the Access Quick RT-PCR system (Promega, Madison, WI) using the following conditions: $45 \mathrm{~min}$ at $48^{\circ} \mathrm{C} ; 1 \mathrm{~min}$ at $94^{\circ} \mathrm{C} ; 25$ cycles: $30 \mathrm{sec}$ at $94^{\circ} \mathrm{C}, 1 \mathrm{~min}$ at $62^{\circ} \mathrm{C}, 1 \mathrm{~min}$ at $72^{\circ} \mathrm{C} ; 6 \mathrm{~min}$ at $72^{\circ} \mathrm{C}$; and a final hold at $4^{\circ} \mathrm{C}$. Primers used for mouse PRLR were $5^{\prime}$ GGCCCCAATTCCTGTTTCTTTAGC-3' and 5'-GCCACTG CCCAGACCATAATCAAAC-3'; primers for glyceraldehyde3-phosphate dehydrogenase $(\mathrm{G} 3 \mathrm{PDH})$ were 5'-ACCACAGT CCATGCCATCAC-3' and 5'-TCCACCACCCTGTTGCT GTA-3'. To ensure no DNA contamination, separate reactions were run without the reverse transcriptase.

McNeuA allograft experiment. McNeuA cells were kindly provided by Dr Michael Campbell, and cultured as previously described (25). At time of implantation, cells were trypsinized, washed with growth medium, counted and re-suspended at a concentration of $3 \times 10^{6}$ cells $/ 0.1 \mathrm{ml}$ in PBS. The mixture was diluted 1:1 (v/v) with Matrigel (BD Biosciences, Franklin Lakes, NJ) and injected into the anterior fat pad of 3-month old homozygous MMTV-neu virgin female mouse $(0.2 \mathrm{ml} /$ mouse). Mice were randomly sorted into 9 groups of 4 mice and treatments with the fusion proteins were initiated three days after implantation.

Mice were intraperitoneally (i.p.) administered a low dose of each fusion protein individually (50 $\mu \mathrm{g} \mathrm{G129R-Endostatin,}$ $50 \mu \mathrm{g}$ G129R-IL2, or $10 \mu \mathrm{g}$ G129R-PE ${ }_{38}$ KDEL), a high dose of each fusion protein individually (100 $\mu \mathrm{g} \mathrm{G129R-Endostatin,}$ $100 \mu \mathrm{g} \mathrm{G} 129 \mathrm{R}-\mathrm{IL} 2$, or $\left.25 \mu \mathrm{g} \mathrm{G} 129 \mathrm{R}-\mathrm{PE}_{38} \mathrm{KDEL}\right)$, the combination of the low doses, the combination of the high doses, or PBS. All dosages were adjusted to a single $0.2 \mathrm{ml}$ injection volume. Dosages were determined from previous published data. These dosages were non-lethal and showed ample difference in growth of T-47D xenografts in nude mice $(12,13)$. Treatments were administered every three days for 27 days. The short (S) and long (L) axes of the tumors were measured and the tumor volume (V) was calculated using the formula for a spheroid ( $\mathrm{V}=4 / 3 \pi \times L / 2 \times S / 2 \times S / 2)$. On day 28 of the experiment, tumors were dissected and split into thirds: one part for fixation in formalin, one part for flow cytometry and one part for cryopreservation. Spleens were removed for flow cytometry. Lungs were removed and stained in Bouin's Fixative (Sigma-Aldrich). The presence or 
absence of macrometastases was recorded and the $\chi^{2}$ test of independence was used to determine significance between treatment groups.

MMTV-neu transgenic mouse mammary tumor surgical model. Due to natural variation in tumor formation rates, mammary tumors from 6 to 10 MMTV-neu mice were removed in intervals based upon similarity in tumor size. Healthy mice showing no ill effects from the surgery were randomized into control and treatment groups. The day after surgery, mice were started on a twice weekly low dose combination regimen (100 $\mu \mathrm{g} \mathrm{G129R-Endostatin,} 100 \mu \mathrm{g}$

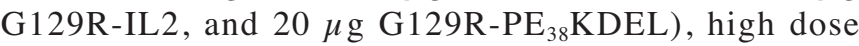
combination regimen (200 $\mu \mathrm{g}$ G129R-Endostatin, $200 \mu \mathrm{g}$ G129R-IL2, and $25 \mu \mathrm{g}$ G129R-PE ${ }_{38}$ KDEL), $200 \mu \mathrm{g}$ G129R 5 days per week, or PBS. Animals were palpitated at time of injections and tumor recurrence was noted; treatments were ceased after recurrent tumors reached $\sim 1 \mathrm{~cm}$ in diameter at which point animals were sacrificed and handled as described above. Kaplan-Meier survival analysis was performed on tumor recurrence utilizing new tumor formation as the end-point. Log-rank analysis was performed to determine significance between individual comparisons and the overall experiment.

Flow cytometry. Single cell suspensions were obtained from the isolated spleens and allograft fractions. The re-suspended splenocytes were diluted 1:10 to $1: 20$ in Guava ${ }^{\circledR}$ ViaCount $^{\circledR}$ Reagent (Guava Technologies, Hayward, CA) which contains two DNA-binding dyes, one of which selectively penetrates apoptotic and dead cells characterized by compromised membrane integrity, and one which penetrates all nucleated cells. Following the 5-min incubation, the stained cells were analyzed on a Guava Personal Cytometer (Guava Technologies). Each sample was analyzed using Guava CytoAnalysis software (Guava Technologies). The total cellular population was determined for each sample and results are reported as averages and SEM of each group. The single cell suspensions from the allografts were enriched for tumor-infiltrating lymphocytes by Percoll (Sigma-Aldrich) density gradient centrifugation. Tumor-infiltrating lymphocytes were collected at the $1.055-1.07 \mathrm{~g} / \mathrm{ml}$ interface and washed three times with PBS. As per guidelines from Guava Technologies, cells were incubated with $5 \mu \mathrm{g} / \mathrm{ml}$ of antibodies directed against CD3 and CD4 or CD3 and CD8. All antibodies were obtained in conjugated form from BD Bioscience (San Jose, CA). Statistical differences between the groups were determined by t-test.

Immunohistochemistry. Formalin-fixed tumors were paraffinembedded, cut into 5- $\mu \mathrm{m}$ sections and placed on glass slides. The sections were deparaffinized in xylene and rehydrated in a graded series of ethanol. The sections were initially covered with hematoxylin for 10-60 sec and washed with water. The sections were then covered by eosin for $60 \mathrm{sec}$, washed with water and dehydrated with ethanol. The slides were placed in xylene before securing a cover slip. For antigen retrieval, deparaffinized and rehydrated slides were submerged in high $\mathrm{pH}$ antigen retrieval solution (Biocare Medical, Concord, CA), placed under pressure in a decloaking chamber
A

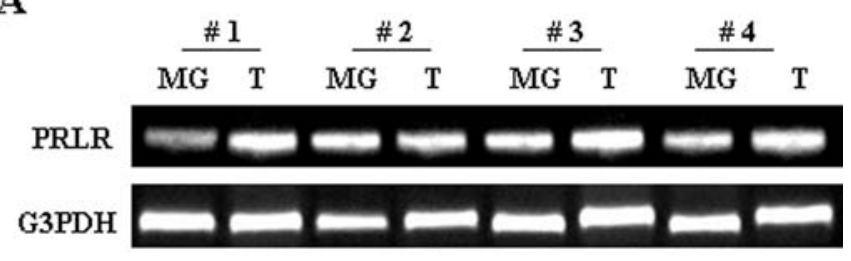

B

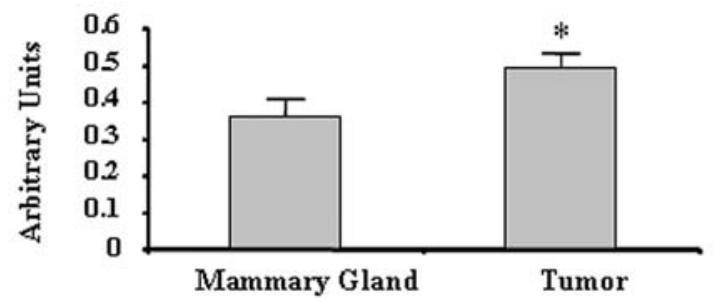

Figure 1. PRLR expression in the surgical model. (A) Comparison of the expression levels of PRLR of the mammary tumors and its adjacent normal mammary gland tissue from 9-month old female mice $(n=4)$ using RT-PCR. PCR products were resolved by electrophoresis in $1 \%$ agarose gels. In data not shown, the reactions were prepared without reverse transcriptase to ensure the product observed was not from DNA contamination. (B) Gel electrophoresis photography was analyzed with the Kodak 1D Image Analysis software to compare the mean intensity of the bands. A statistical difference was observed between the mammary gland and tumor tissue $\left({ }^{*} \mathrm{P}<0.02\right)$.

(Biocare Medical), and washed with distilled water. Slides were blocked for $10 \mathrm{~min}$ and incubated with anti-CD4 (BC/1F6, Biocare Medical) or anti-CD31 (MEC13.3, Biocare Medical) for 60 and $45 \mathrm{~min}$, respectively, and detected using the Evision ${ }^{+}$ Dual Link System-HRP detection kit (Dako, Carpinteria, CA). Antibody/enzyme complex was visualized with DAB (Biocare Medical) for 3 min and counterstained with hematoxylin for $5 \mathrm{~min}$.

\section{Results}

PRLR expression levels in normal or malignant mammary tissue of MMTV-neu mice. PRLR expression levels in tumors and normal mammary gland of MMTV-neu mice were compared by RT-PCR. As shown in Fig. 1, expression levels of mouse PRLR in the tumors were significantly increased $(\mathrm{P}<0.05)$ as compared to that of adjacent normal mammary gland, providing a rationale for targeted treatment with the PRLR antagonist based fusion proteins. In data not shown, the reactions were run without reverse transcriptase to ensure the product observed was not from DNA contamination.

McNeuA allograft studies. The tumor growth rate of all treatment groups was retarded by day 24 of the study as compared to that of the PBS controls (Fig. 2A, low dose; and Fig. 2B, high dose). As shown in Fig. 2A, a significant difference in the tumor volumes was observed for both G129R-PE ${ }_{38}$ KDEL monotherapy and the combination $(\mathrm{P}<0.001$ in both cases $)$. While a decrease in tumor volume was observed in the G129R-IL2 and G129R-Endostatin treatment groups, these differences were not significant. In the high dose treatments groups there was also a significant 
A

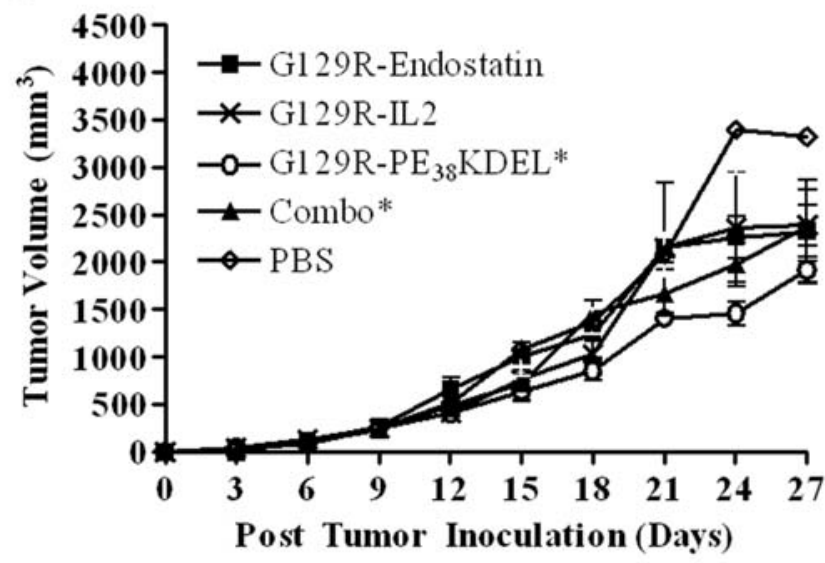

B

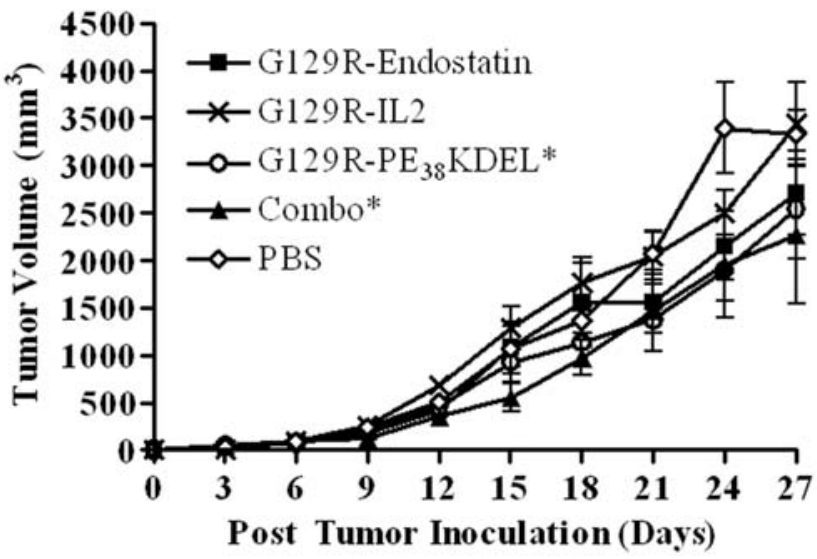

Figure 2. Tumor growth in McNeuA cell allograft studies. After tumor cell inoculation, two dosages of each fusion protein and two doses of combination were used to evaluate efficacy. The low combination dosages (A) were composed of G129R-Endostatin $(50 \mu \mathrm{g})$, G129R-IL2 $(50 \mu \mathrm{g})$ and G129R$\mathrm{PE}_{38} \mathrm{KDEL}(10 \mu \mathrm{g})$ and the high dose combination (B) composed of G129R-Endostatin $(100 \mu \mathrm{g})$, G129R-IL2 $(100 \mu \mathrm{g})$ and G129R-PE ${ }_{38}$ KDEL $(25 \mu \mathrm{g})$ administered as a single injection. All treatments were diluted in $0.2 \mathrm{ml}$ PBS and injected i.p. into the mouse at the specified interval. Tumor volumes were calculated at each treatment time-point throughout the study. A significant decrease in tumor volume was observed in the final two measurements of the both individual G129R-PE ${ }_{38} \mathrm{KDEL}$ dosages as well as both combination treatments as compared to the PBS control $(\mathrm{P}<0.001$ in all cases).

reduction in tumor volume in the G129R-PE ${ }_{38} \mathrm{KDEL}$ and combination treatment groups $(\mathrm{P}<0.001$ in both cases $)$. No significant difference in tumor volume was observed between the groups receiving low or high dose combination treatments.

To examine the IL2-like activity of G129R-IL2, as well as determine any signs of global toxicity, tumor infiltrating lymphocytes and splenic cellularity were examined by flow cytometry. In comparison to the PBS control group, a significant increase $(\mathrm{P}<0.05)$ in the percent of $\mathrm{CD}^{+}$cells (Fig. 3A) was observed within the tumors of the mice receiving the high-dose combination treatment. No significant difference was observed in cells labeled with anti-CD4 or anti-CD3 (data not shown). There was no significant alteration in the total cellularity within the spleens of either of the combination groups when compared to the PBS control groups (Fig. 3B). No alterations were observed in body weight, physical appearance,
A

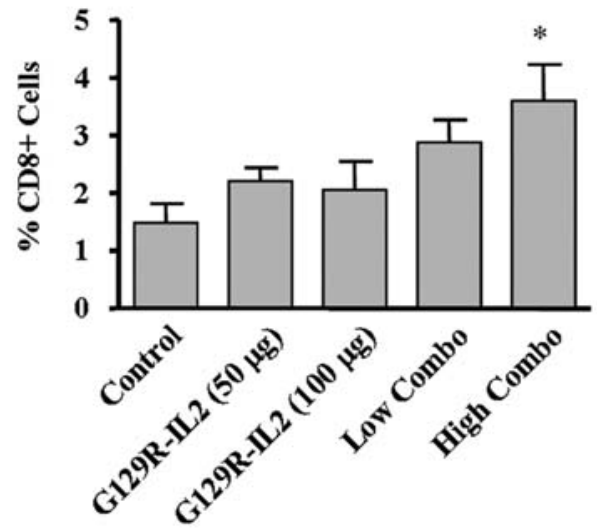

B

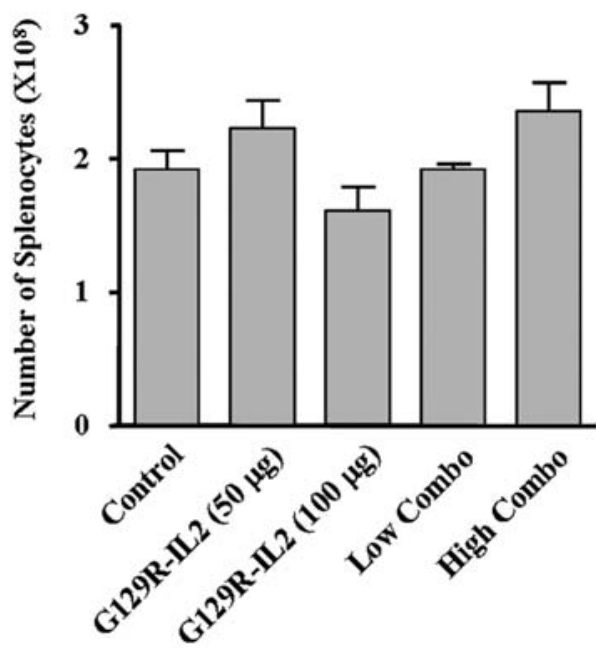

Figure 3. T-cell infiltration in McNeuA cell allograft studies. After tumor cell inoculation, two dosages of G129R-IL2 (50 or $100 \mu \mathrm{g})$ and two doses of combination were used to evaluate efficacy. The low combination dosage was composed of G129R-Endostatin $(50 \mu \mathrm{g})$, G129R-IL2 $(50 \mu \mathrm{g})$ and G129R-PE ${ }_{38} \mathrm{KDEL}(10 \mu \mathrm{g})$ and the high dose combination composed of G129R-Endostatin $(100 \mu \mathrm{g})$, G129R-IL2 $(100 \mu \mathrm{g})$ and G129R-PE ${ }_{38} \mathrm{KDEL}$ $(25 \mu \mathrm{g})$ administered as a single injection. All treatments were diluted in $0.2 \mathrm{ml}$ PBS and injected i.p. into the mouse at the specified interval. At the end of experiment, the tumors were dissected, tumor infiltrating lymphocytes were isolated by density centrifugation and the total $\mathrm{CD} 8^{+}$tumor infiltrating lymphocytes were assessed by flow cytometry (A), results are expressed as a percent of total cells in gradient. The splenocytes were also enumerated and no difference was observed upon treatment with G129R-IL2 containing regimen $(\mathrm{B})$.

or behavior (data not shown).

MMTV-neu transgenic mouse mammary tumor surgical model studies. A novel and more clinically relevant mouse tumor model was developed for this study. Naturally occurring tumors were surgically removed from MMTV-neu mice. Surgery was followed with adjuvant treatment to thwart tumor recurrence. Based upon the results from McNeuA allograft study, two different combination regimens were used in the surgical tumor model study. After surgical removal of the primary tumor, the mice were treated with PBS, G129R, three individual fusion proteins as a monotherapy, or combination of the three G129R fusion proteins at low or high doses. Kaplan-Meier survival analysis was performed using tumor recurrence as the end-point (Fig. 4). The overall significance of the study, when all treatment groups were included, was found to be $\mathrm{P}<0.004$.

The median values for tumor recurrence were doubled in 
A

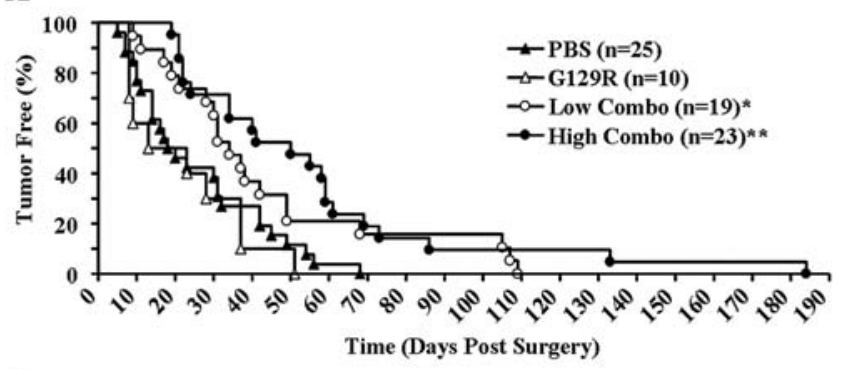

B

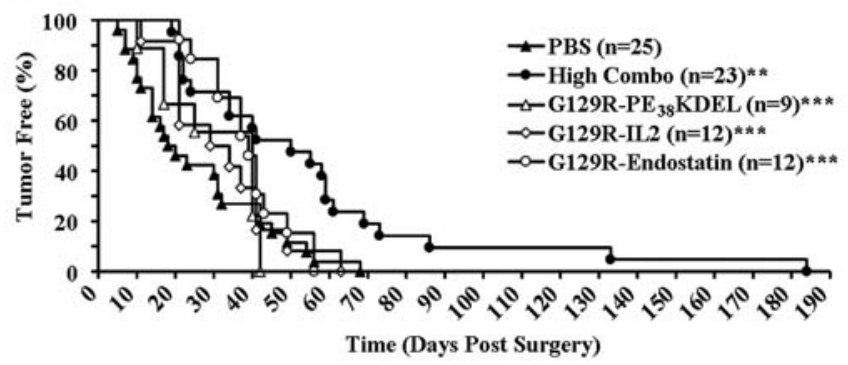

C

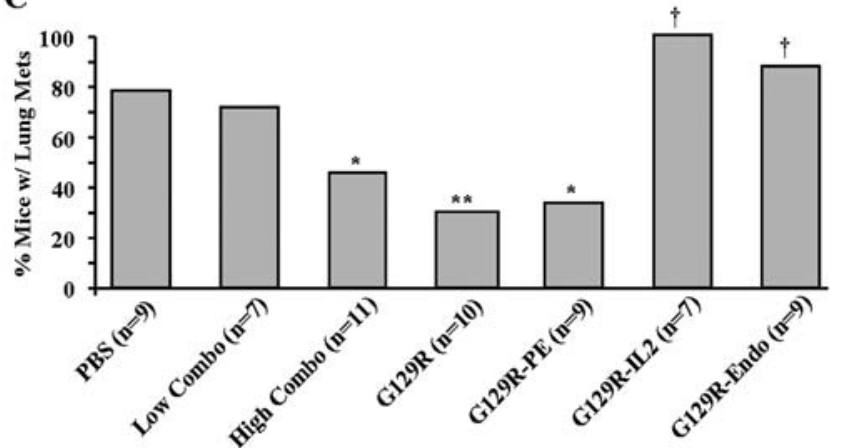

Figure 4. Kaplan-Meier survival analysis of the MMTV-neu transgenic mouse mammary tumor surgical study. Tumor recurrence was used as the end-point for this analysis. (A) Median tumor recurrence for PBS controls was 17 days, G129R 18 days, low combination 34 days, and high combination 52.5 days. The low $\left({ }^{*} \mathrm{P}<0.02\right)$ and high combination $\left({ }^{* *} \mathrm{P}<0.002\right)$ significantly delayed the recurrence for tumors in this model. (B) Median tumor recurrence for G129R-PE ${ }_{38}$ KDEL was 40 days, G129R-IL2 31.5 days, and G129REndostatin 39 days. Each component was significantly different from both PBS and the high combination $\left({ }^{* * *} \mathrm{P}<0.02\right)$. Log-rank analysis of the survival curve revealed a highly significant difference between the treated groups and the control $(\mathrm{P}<0.004)$. (C) Total lung metastasis. Lungs were excised at sacrifice and fixed in Bouin's fixative to observe metastasis. The percentages of animals with lung metastases are reported and the $\chi^{2}$ test for independence was utilized to determine significance. The high combination, G129R, and G129R-PE ${ }_{38}$ KDEL were significantly reduced in comparison to the PBS treated group $\left({ }^{*} \mathrm{P}<0.1,{ }^{* *} \mathrm{P}<0.05\right)$. Both the G129R-IL2 and G129R-Endostatin fusion alone were significantly higher then the high combination $\left({ }^{\dagger} \mathrm{P}<0.05\right)$.

the low combination treatment group (34 days) and nearly tripled in the high combination treatment group (50 days) (Fig. 4A), each resulting in a highly significant delay, $\mathrm{P}<0.02$ and 0.0002 , respectively. G129R-PE ${ }_{38} \mathrm{KDEL}$ (40 days), G129R-IL2 (31.5 days), and G129R-Endostatin (39 days) significantly $(\mathrm{P}<0.02)$ delayed the median values relative to PBS control (Fig. 4B). Also, tumor recurrence in the high combination treatment group was significantly delayed as compared to the individual fusion protein treatment groups $(\mathrm{P}<0.05)$. Log-rank analysis of the survival data revealed a significant difference between the curves.

Metastasis to the lungs was reduced in a manner consistent with a dose-dependent effect by the combination treatments; the high dose combination treatment group developed lung metastases only $40 \%$ of the time, compared to $78 \%$ for PBS (Fig. 4C). This reduction was also significantly lowered by G129R-PE ${ }_{38} \mathrm{KDEL}$ (30\%). It is noteworthy that although there was no difference between the G129R treatment group (18 days) and the PBS control group (17 days) in median values for tumor recurrence (Fig. 4A), there was a significant reduction $(33 \%)$ in lung metastases in the group receiving G129R treatment as compared to PBS control. All other individual treatments showed no significant reduction in lung metastases. The $\chi^{2}$ test for independence determined that the high combination, G129R, and G129R-PE ${ }_{38} \mathrm{KDEL}$ treated groups differed significantly from the PBS group and that the G129R-IL2 and G129R-Endostatin receiving groups differed from the high combination treated group. There was no appreciable difference between the age of mice at time of surgery and the mass of tumors removed nor were there any signs of toxicity, i.e., no alteration in body weight, appearance or behavior.

Specific biomarkers were examined in secondary tumor samples to analyze the specific efficacy of the fusion proteins. Since G129R-Endostatin fusion protein was designed to serve as a targeted angiogenesis inhibitor, the blood vessel density (measured by CD31 level, an endothelial cell marker) within the recurrent tumors was examined. An obvious decrease in CD31-positive cells was observed in all treatment groups when compared to the PBS group, suggesting a reduced vessel density within the tumors (Fig. 5A). There was no significant difference between the treatment groups. To examine response to the G129R-IL2 fusion protein, tumor sections were stained for CD4, a marker of $\mathrm{T}$ helper cells. There was a remarkable increase in the number of CD4-positive cells observed in the treatment groups when compared to the PBS control group (Fig. 5B).

\section{Discussion}

Conventional cancer treatment begins with radiation therapy or small molecules that target DNA synthesis and replication activities. Treatment is usually followed by surgical removal of the shrunken mass. After surgery, patients typically receive additional rounds of radiation and/or chemotherapy (26). While there have been vast improvements in the equipment for delivering radiation therapy (27) and new methods of administering the chemotherapy (28), these methods often damage many non-cancerous cells due to a lack in specificity, thereby resulting in significant adverse side-effects. In the past 10-15 years the paradigm of cancer chemotherapy has been shifted towards designing so-called 'smart molecules' that have ability to selectively target cancer cells. Efforts have aimed at identifying and targeting unique markers of cancer cells such as over-expressed receptors, tumor antigens, or unique biological features of a tumor (29). Therapeutics targeting breast cancer based on receptor status have yielded a wide array of options for treatment of the disease including selective estrogen receptor (ER) modulators (SERMs) for ER-positive cancers (30) and trastuzumab (16) or lapatinib (31) for HER2-positive cancers. Over-expression of PRLR in breast cancer led to the development of a PRLR antagonist, G129R, and G129R based fusion proteins (12-14). 
A

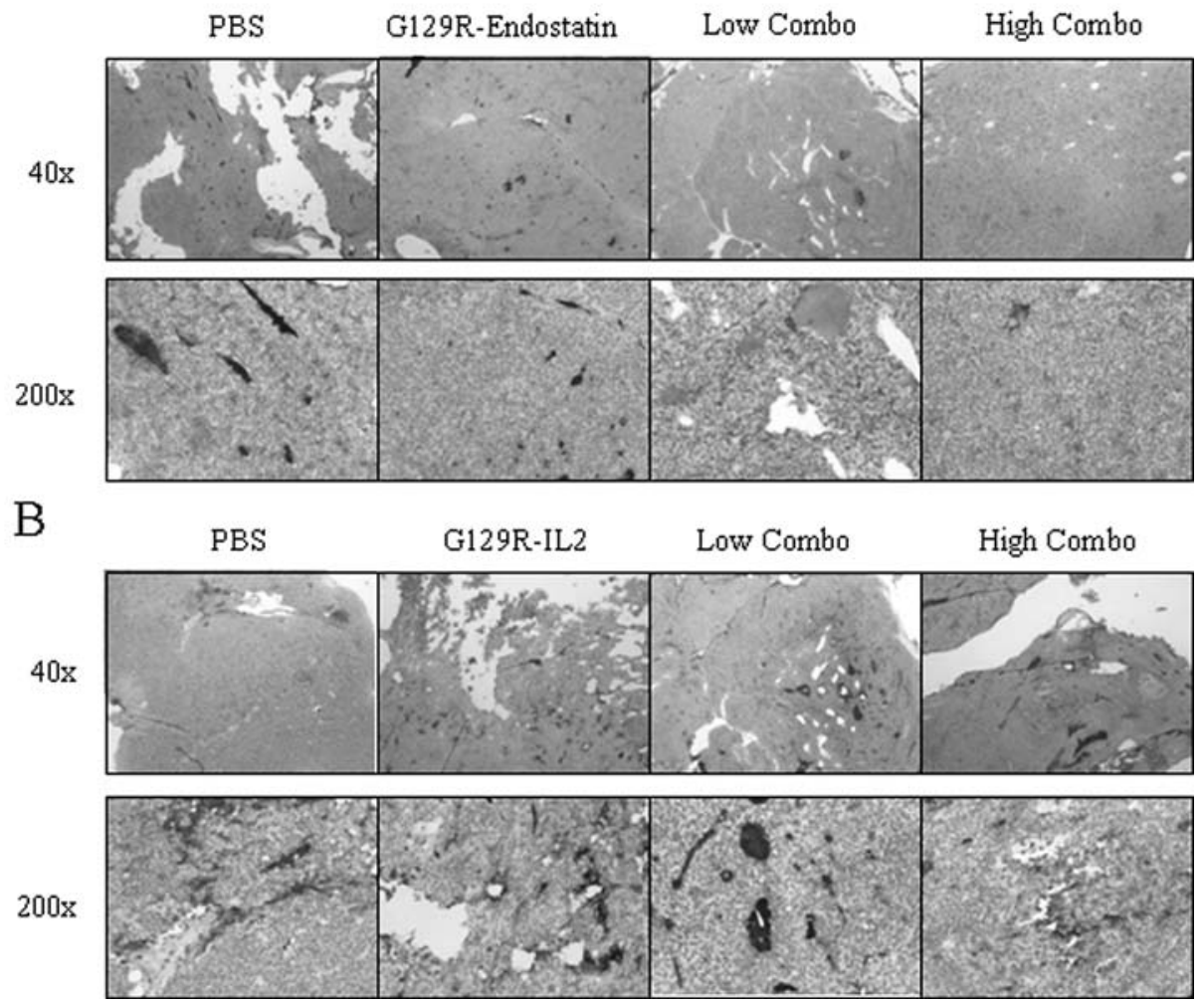

Figure 5. MMTV-neu transgenic mouse mammary tumor surgical study biomarkers. (A) Tumor vessel density. Representative sections of tumors from PBS controls, G129R-Endostatin, and low and high combinations tumors after treatment were stained for CD31. Vessel density was remarkably lower after primary tumor removal and treatment with the fusion protein combinations or G129R-Endostatin alone. (B) Tumor infiltrating lymphocytes. Representative sections of tumors from PBS controls, G129R-IL2, and low and high combinations tumors after treatment were stained for CD4. The treatment regimens were able to increase the $\mathrm{CD} 4^{+}$cells within the tumors after primary tumor removal and treatment with the fusion protein combinations or G129R-Endostatin alone. Magnification, top of each panel x40 and bottom of each panel x200.

Another important trend in cancer therapy has been to develop effective combinatorial approaches. Strategies for combining chemotherapies that have proven effective as a single agent fall into at least three categories. First of which is to use agents that attack the same oncoprotein via different mechanisms. For example, trastuzumab and lapatinib or trastuzumab and pertuzumab have been used to treat HER2positive breast cancer (16) and cetuximab with gefitinib or erlotinib to treat EGFR-over-expressing tumors (15). A second approach to maximize the efficacy of combination therapy is to attack multiple targets present in same oncogenic signaling cascade. For example, the src homology domain has been shown to have a critical role in HER2 signaling and is believed to be an important factor for metastasis of HER2 overexpressing cancers (32). The use of dasatinib, a small molecule inhibitor of src has proven beneficial to HER2-positive breast cancer when used in combination with trastuzumab (33). Furthermore, use of trastuzumab in combination with the PRLR antagonist, G129R, has also been shown to have an additive inhibitory effect on HER2 and MAPK phosphorylation, and the proliferation of T-47D and BT-474 breast cancer cells both in vitro and in vivo (4). Finally, attacking multiple hallmarks of cancer simultaneously using various cancer therapeutics is under investigation. An example of this approach would be use of trastuzumab, which targets the oncogene HER2, in combination with bevacizumab, which targets tumor angiogenesis, which has been demonstrated effective in small cell lung caner (17).

In this study, the efficacy of using three fusion proteins (G129R-IL2, G129R-PE ${ }_{38}$ KDEL, G129R-Endostatin) to target breast cancer and multiple hallmarks of cancer was examined. The in vivo capabilities of two of these fusion proteins has been previously tested using breast cancer cell xenograft model $(12,13)$. These results are further confirmed in this study (Fig. 2) utilizing the McNeuA mammary cancer cell line derived from an MMTV-neu mouse tumor (25). The McNeuA allograft study laid the foundation for optimization of fusion-protein dosages in our surgical model. The data presented here are also the first demonstration of the function of the second moiety of the fusion proteins in vivo. The T-cell infiltration in the allograft and surgical models (Figs. 3A and 5B) demonstrate the ability of peripherally administered G129R-IL2 to enhance the immune response to the tumor without causing serious systemic immune reaction, as evidenced by the lack of increased cellularity in the spleens (Fig. 3B). The decrease in blood vessel density within the tumors of the surgical model indicates (Fig. 5A) that G129REndostatin can limit angiogenesis in vivo.

Although models utilizing human cancer cells implanted into athymic mice have been widely used and proven to be a valuable tool in drug testing, these models have major drawbacks; in particular, they lack the host immune system 
influence on the tumor. In addition, xenograft models do not characterize natural tumor developmental processes. Therefore, the surgical mouse tumor model presented here may prove to be more clinically relevant since it simulates a clinical setting, i.e., therapies applied after surgical removal of naturally occurring primary tumors. The efficacy of the therapy is not measured by slowing the growth of tumor but by the tumor recurrence time/rate and metastasis index.

The data presented here demonstrate that the G129R fusion proteins significantly delayed tumor recurrence (Fig. 4A and B), reduced lung metastasis (Fig. 4C), decreased the blood vessel density within the tumor (Fig. 5A), and increased the quantity of $\mathrm{CD}^{+}$cells within the recurrent tumors (Fig. 5B). There was a highly significant and dosage-dependent delay in tumor recurrence when comparing the PBS control to either combination group (Fig. 4A). If the life expectancy of a human female (34) is compared with that of the MMTV-neu mouse (35), an average of 17 days post-surgery tumor-free phase in the control group translates to approximately 3-5 years in a human. Remarkably, the low dose combination treatment resulted in a 2-fold increase in the tumor-free phase (34 days). The tumor recurrence rate in the high combination treated group was one-third that of the control group.

Metastasis of the tumor to a distant site is typically the leading cause for the deterioration of the patient and ultimately determines the patient's ability to survive after surgical removal. In our study model, both the low and high dosages of the combination therapies yielded $>50 \%$ reduction in total lung metastasis lesions, which directly translates to total survival time. The ability to measure distant metastasis further demonstrates the clinical relevancy and utility for the novel surgical model presented herein. The difference observed in total lung metastases in response to the three fusion proteins as monotherapy (Fig. 4C) may be attributed to the function of the second moiety of each fusion protein. The cytotoxic nature of the G129R-PE ${ }_{38} \mathrm{KDEL}$ allows it to elicit its effect directly on tumor cells, while both G129R-IL2 and G129REndostatin were designed to target tumor hallmarks such as host immune system and tumor angiogenesis in addition to the tumor cells. Therefore, one can speculate that G129R$\mathrm{PE}_{38} \mathrm{KDEL}$ is more effective at decreasing circulating PRLRpositive tumor cells, thereby reducing metastasis, and G129RIL2 and G129R-Endostatin fusion is more effective on established tumors.

It is noteworthy that G129R monotherapy has minimal effect both in vitro (data not shown) and in the surgical model (Fig. 4A) measured by tumor recurrence as compared to the PBS control group. However, G129R was surprisingly effective at preventing lung metastasis in the surgical model (Fig. 4C). As a matter of fact, daily G129R treatment was as effective as the twice weekly high dose combination treatment, which further confirms the therapeutic potential of G129R in HER2-positive breast cancer.

In conclusion, this report presents a novel approach utilizing G129R-fusion proteins to specifically target multiple hallmarks of cancer. This combination therapy proved to be significantly effective at reducing tumor growth as well as tumor recurrence and metastasis. Further, this report presents a new method for the testing of therapeutic strategies in a clinically relevant setting.

\section{Acknowledgements}

The authors would like to thank Faye Bryson and Pathology Consultants of Greenville, SC for their time in preparing the materials for the labeling of the IHC(P) sections with CD4 and CD31. We would also like to thank Guillermo De Angulo for his assistance with the mouse surgeries and dosing. This work was supported in part by the Endowment Fund of the Greenville Hospital System and grants from the Susan G. Komen Foundation (BCTR0402985 and BCTR091306).

\section{References}

1. Reynolds C, Montone KT, Powell CM, Tomaszewski JE and Clevenger CV: Expression of prolactin and its receptor in human breast carcinoma. Endocrinology 138: 5555-5560, 1997.

2. Touraine P, Martini JF, Zafrani B, Durand JC, Labaille F, Malet C, Nicolas A, Trivin C, Postel-Vinay MC, Kuttenn F and Kelly PA: Increased expression of prolactin receptor gene assessed by quantitative polymerase chain reaction in human breast tumors versus normal breast tissues. J Clin Endocrinol Metab 83: 667-674, 1998.

3. Ormandy CJ, Hall RE, Manning DL, Robertson JF, Blamey RW, Kelly PA, Nicholson RI and Sutherland RL: Coexpression and cross-regulation of the prolactin receptor and sex steroid hormone receptors in breast cancer. J Clin Endocrinol Metab 82: 3692-3699, 1997.

4. Scotti ML, Langenheim JF, Tomblyn S, Springs AE and Chen WY: Additive effects of a prolactin receptor antagonist, G129R, and herceptin on inhibition of HER2-overexpressing breast cancer cells. Breast Cancer Res Treat 111: 241-250, 2008.

5. Yamauchi T, Yamauchi N, Ueki K, Sugiyama T, Waki H, Miki H, Tobe K, Matsuda S, Tsushima T, Yamamoto T, Fujita T, Taketani Y, Fukayama M, Kimura S, Yazaki Y, Nagai $R$ and Kadowaki T: Constitutive tyrosine phosphorylation of ErbB-2 via Jak2 by autocrine secretion of prolactin in human breast cancer. J Biol Chem 275: 33937-33944, 2000.

6. Chen WY, Ramamoorthy P, Chen N, Sticca R and Wagner TE: A human prolactin antagonist, hPRL-G129R, inhibits breast cancer cell proliferation through induction of apoptosis. Clin Cancer Res 5: 3583-3593, 1999.

7. Ropero S, Menendez JA, Vazquez-Martin A, Montero S, Cortes-Funes $\mathrm{H}$ and Colomer R: Trastuzumab plus tamoxifen: anti-proliferative and molecular interactions in breast carcinoma. Breast Cancer Res Treat 86: 125-137, 2004.

8. Goffin V and Kelly PA: Prolactin and growth hormone receptors. Clin Endocrinol (Oxford) 45: 247-255, 1996.

9. Beck MT, Peirce SK and Chen WY: Regulation of bcl-2 gene expression in human breast cancer cells by prolactin and its antagonist, hPRL-G129R. Oncogene 21: 5047-5055, 2002.

10. Cataldo L, Chen NY, Yuan Q, Li W, Ramamoorthy P, Wagner TE, Sticca RP and Chen WY: Inhibition of oncogene STAT3 phosphorylation by a prolactin antagonist, hPRLG129R, in T-47D human breast cancer cells. Int J Oncol 17: 1179-1185, 2000.

11. Peirce SK and Chen WY: Human prolactin and its antagonist, hPRL-G129R, regulate bax and bcl-2 gene expression in human breast cancer cells and transgenic mice. Oncogene 23: 1248-1255, 2004.

12. Beck MT, Chen NY, Franek KJ and Chen WY: Prolactin antagonist-endostatin fusion protein as a targeted dual-functional therapeutic agent for breast cancer. Cancer Res 63: 3598-3604, 2003.

13. Zhang G, Li W, Holle L, Chen N and Chen WY: A novel design of targeted endocrine and cytokine therapy for breast cancer. Clin Cancer Res 8: 1196-1205, 2002.

14. Langenheim JF and Chen WY: Development of a prolactin receptor-targeting fusion toxin using a prolactin antagonist and a recombinant form of Pseudomonas exotoxin A. Breast Cancer Res Treat 90: 281-293, 2005.

15. Harari PM: Epidermal growth factor receptor inhibition strategies in oncology. Endocr Relat Cancer 11: 689-708, 2004.

16. Pal SK and Pegram M: HER2 targeted therapy in breast cancer... beyond Herceptin. Rev Endocr Metab Disord 8: 269-277, 2007. 
17. Thatcher N: The place of targeted therapy in the patient management of non-small cell lung cancer. Lung Cancer 57 (Suppl. 2): S18-S23, 2007.

18. Safran H, Dipetrillo T, Akerman P, Ng T, Evans D, Steinhoff M, Benton D, Purviance J, Goldstein L, Tantravahi U and Kennedy T: Phase I/II study of trastuzumab, paclitaxel, cisplatin and radiation for locally advanced, HER2 overexpressing, esophageal adenocarcinoma. Int J Radiat Oncol Biol Phys 67: 405-409, 2007.

19. Quick QA and Gewirtz DA: Enhancement of radiation sensitivity, delay of proliferative recovery after radiation and abrogation of MAPK (p44/42) signaling by imatinib in glioblastoma cells. Int J Oncol 29: 407-412, 2006.

20. Huang S, Armstrong EA, Benavente S, Chinnaiyan P and Harari PM: Dual-agent molecular targeting of the epidermal growth factor receptor (EGFR): combining anti-EGFR antibody with tyrosine kinase inhibitor. Cancer Res 64: 53555362, 2004

21. Matar P, Rojo F, Cassia R, Moreno-Bueno G, Di Cosimo S, Tabernero J, Guzman M, Rodriguez S, Arribas J, Palacios J and Baselga J: Combined epidermal growth factor receptor targeting with the tyrosine kinase inhibitor gefitinib (ZD1839) and the monoclonal antibody cetuximab (IMC-C225): superiority over single-agent receptor targeting. Clin Cancer Res 10: 6487-6501, 2004.

22. Boone T, Chazin V, Kenney W, Swanson E and Altrock B Construction, purification and biological activities of recombinant human interleukin-2 analogs. Dev Biol Stand 69: 157-168, 1988.

23. Kreitman RJ, Siegall CB, Chaudhary VK, FitzGerald DJ and Pastan I: Properties of chimeric toxins with two recognition domains: interleukin 6 and transforming growth factor alpha at different locations in Pseudomonas exotoxin. Bioconjug Chem 3: 63-68, 1992 .

24. Muller WJ, Arteaga CL, Muthuswamy SK, Siegel PM Webster MA, Cardiff RD, Meise KS, Li F, Halter SA and Coffey RJ: Synergistic interaction of the Neu proto-oncogene product and transforming growth factor alpha in the mammary epithelium of transgenic mice. Mol Cell Biol 16: 5726-5736, 1996.

25. Campbell MJ, Wollish WS, Lobo M and Esserman LJ: Epithelial and fibroblast cell lines derived from a spontaneous mammary carcinoma in a MMTV/neu transgenic mouse. In Vitro Cell Dev Biol Anim 38: 326-333, 2002.
26. Carlson RW, Anderson BO, Burstein HJ, Carter WB, Edge SB, Farrar WB, Goldstein LJ, Gradishar WJ, Hayes DF, Hudis CA, Jahanzeb M, Ljung BM, Kiel K, Marks LB, McCormick B, Nabell LM, Pierce LJ, Reed EC, Silver SM, Smith ML, Somlo G, Theriault RL, Ward JH, Winer EP and Wolff AC: Invasive breast cancer. J Natl Compr Cancer Netw 5: 246-312, 2007.

27. Romanelli P, Schaal DW and Adler JR: Image-guided radiosurgical ablation of intra- and extra-cranial lesions. Technol Cancer Res Treat 5: 421-428, 2006.

28. Kim KY: Nanotechnology platforms and physiological challenges for cancer therapeutics. Nanomedicine 3: 103-110, 2007.

29. Hanahan D and Weinberg RA: The hallmarks of cancer. Cell 100: 57-70, 2000.

30. Jordan VC: Antiestrogenic and antitumor properties of tamoxifen in laboratory animals. Cancer Treat Rep 60: 14091419, 1976

31. Bilancia D, Rosati G, Dinota A, Germano D, Romano R and Manzione L: Lapatinib in breast cancer. Ann Oncol 18 (Suppl. 6): vi26-30, 2007.

32. Schade B, Lam SH, Cernea D, Sanguin-Gendreau V, Cardiff RD, Jung BL, Hallett M and Muller WJ: Distinct ErbB-2 coupled signaling pathways promote mammary tumors with unique pathologic and transcriptional profiles. Cancer Res 67: 7579-7588, 2007.

33. Finn RS, Dering J, Ginther C, Wilson CA, Glaspy P, Tchekmedyian $\mathrm{N}$ and Slamon DJ: Dasatinib, an orally active small molecule inhibitor of both the src and abl kinases, selectively inhibits growth of basal-type/'triple-negative' breast cancer cell lines growing in vitro. Breast Cancer Res Treat 105: 319-326, 2007.

34. Minino AM, Heron MP and Smith BL: Deaths: preliminary data for 2004. Natl Vital Stat Rep 54: 1-49, 2006

35. Anisimov VN, Arbeev KG, Popovich IG, Zabezhinksi MA, Arbeeva LS and Yashin AI: Is early life body weight a predictor of longevity and tumor risk in rats? Exp Gerontol 39: 807-816, 2004. 